\title{
P I 6-54 LB. Blood CCR6+ Th I 7 and Th I Th I 7 but not CCR6neg Th I cells are targets for HIV replication and their frequency is diminished in HIV-infected subjects
}

\author{
A Gosselin 4 , P Monteiro ${ }^{4}$, N Chomont ${ }^{4}$, F Diaz-Griffero1, VS Wacleche ${ }^{4}$, \\ EA Said ${ }^{4}$, S Fonseca ${ }^{4}$, M El-Far ${ }^{4}$, M Boulassel $^{2}$, J Routy ${ }^{3}$, R Sekaly ${ }^{4}$ and \\ P Ancuta*4
}

Address: ${ }^{1}$ Dana-Farber Cancer Institute and Harvard Medical School, Boston, MA, USA, ${ }^{2}$ Division of Hematology, McGill University Health Centre, Montréal, Québec, Canada, ${ }^{3}$ Montréal Chest Institute, McGill University Health Centre, Montréal, Québec, Canada and ${ }^{4}$ Université de Montréal, CHUM-Research Center, and INSERM Unit 743, Montréal, Québec, Canada

* Corresponding author

from AIDS Vaccine 2009

Paris, France. 19-22 October 2009

Published: 22 October 2009

Retrovirology 2009, 6(Suppl 3):P407 doi:10.1 I86/1742-4690-6-S3-P407

This abstract is available from: http://www.retrovirology.com/content/6/S3/P407

(C) 2009 Gosselin et al; licensee BioMed Central Ltd.

\section{Background}

Persistence of HIV in discrete CD4+ T-cell subsets is a barrier toward viral eradication. In an effort to identify primary T-cell subsets that play a critical role in HIV pathogenesis, we investigated the lineage-commitment and susceptibility to HIV infection of CD4+ T-cell subsets identified based on differential expression of the chemokine receptors CCR4, CXCR3, et CCR6.

\section{Methods}

T-cell subsets were sorted from HIV-infected and -uninfected individuals by polychromatic flow cytometry. Expression of lineage-specific transcription factors and cytokines was quantified by real-time RT-PCR and ELISA, respectively. HIV replication and integration were measured by HIV-p24 ELISA and real-time PCR, respectively. The frequency of T-cell subsets was analyzed in HIVinfected and -uninfected individuals.

\section{Results}

CCR4+CXCR3negCCR6+, CCR4+CXCR3negCCR6neg, CCR4negCXCR3+CCR6+, and CCR4negCXCR3+CCR6neg T-cells expressed cytokines and transcription factors specific for Th17, Th2, Th1Th17, and Th1 lineages, respectively. Th17 and Th1Th17 expressed the HIV co-receptors CCR5 and CXCR4 and were permissive to $\mathrm{R} 5$ and $\mathrm{X} 4 \mathrm{HIV}$ replication. Th2 expressed CXCR4 but not CCR5 and were permissive to X4 HIV only. Th1 expressed CCR5 and CXCR4, but were resistant to R5 and X4 HIV in vitro. Th17 and Th1Th17 but not Th1 cells harbored high levels of integrated HIVDNA and their frequency was significantly diminished in HIV-infected subjects under anti-retroviral therapy. Th17 and Th1Th17 selectively produced CCL20 and expressed gut- and lymph node-homing molecules.

\section{Conclusion}

We provide evidence that CCR6+ Th17 and Th1Th17 play a critical role in HIV pathogenesis by an increased permissiveness to HIV infection and ability to infiltrate anatomic sites of viral replication and recruit more CCR6+ T-cells to these sites. We also identified a CCR6neg Th1 subset resistant to HIV infection and depletion in vivo. New therapeutic strategies aimed at HIV eradication should interfere with HIV replication in CCR6+ Th17 and Th1Th17 subsets. 